\title{
Perfil epidemiológico e espacial dos casos novos de hanseníase notificados em Feira de Santana no período de 2005- 2015
}

\section{Epidemiological and spatial profile of new leprosy cases notified at Feira de Santana (Brazil) between 2005 and 2015}

\author{
Jucileide Moreira Alves ${ }^{1}$ \\ Roquenei da Purificação Rodrigues ${ }^{2}$ \\ Monalisa Cristiany Santos Carvalho ${ }^{3}$ (1) \\ 1,3Faculdade Estácio de Feira de Santana (Feira de Santana). Bahia, Brasil. \\ jucileidealves24@hotmail.com, monalisa.cristiny@hotmail.com \\ ${ }^{2}$ Autor para correspondência. Hospital Estadual da Criança (Feira de Santana). Bahia, Brasil. roquenei@gmail.com
}

\begin{abstract}
RESUMO | INTRODUÇÃO: A Hanseníase é uma doença infectocontagiosa de evolução crônica causada pelo Mycrobacterim leprae e representa um problema de saúde pública. OBJETIVOS: Objetivou-se com este estudo descrever o perfil epidemiológico e espacial dos casos novos de hanseníase, notificados no município de Feira de Santana - BA, no período de 2005 a 2015. MÉTODOS: Trata-se de um estudo epidemiológico, descritivo com abordagem quantitativa, em que os dados foram coletados do Sistema de informação de agravos de notificação. RESULTADOS: Entre os anos de 2005-2015 foram notificados 1239 casos novos de hanseníase no município em questão. Destes casos, o maior número se deu entre os indivíduos do sexo masculino e entre os indivíduos pardos. A faixa etária de 35-49 anos e com baixa escolaridade apresentaram a maior ocorrência da doença. Quanto à forma clínica, a dimorfa preponderou. E a maioria das pessoas não apresentou qualquer tipo de incapacidade relacionada à doença. A respeito da distribuição espacial da hanseníase, os bairros que apresentaram o maior número de casos foram: Tomba, Brasília, Calumbi, Gabriela, Campo Limpo, Mangabeira e os distritos rurais, foram Bonfim da Feira e Humildes. CONSIDERAÇÕES FINAIS: A caracterização epidemiológica e a distribuição espacial dos dados tornam-se importante para intensificação de estratégias de promoção e proteção à saúde, a fim de se reduzir a ocorrência da patologia.
\end{abstract}

PALAVRAS-CHAVE: Hanseníase, Incidência, Epidemiologia. Distribuição Espacial.

\begin{abstract}
INTRODUCTION: Leprosy is an infectious disease of chronic evolution caused by Mycobacterium leprae and represents a public health problem. OBJECTIVE: The objective of this study was to describe the epidemiological and spatial profile of new cases of leprosy reported in the municipality of Feira de Santana -BA, from 2005 to 2015. METHODS: This is an epidemiological, descriptive study with a quantitative approach in that the data were collected from the Notification of Injury Information System. RESULTS: Between 2005 and 2015, 1239 new cases of leprosy were reported in the municipality in question. Of these cases, the largest number of cases occurred among males and in both sexes, brown individuals, aged 35-49 years and with low schooling, had the highest occurrence of the disease. As for the clinical form, the dimorph preponderated. Furthermore, most people did not have any disability related to the disease. Regarding the spatial distribution of leprosy, the neighborhoods that presented the largest number of cases were: Tomba, Brasília, Calumbi, Gabriela, Campo Limpo, Mangabeira, and the rural districts were Bonfim da Feira and Humildes. FINAL REMARKS: The epidemiological characterization and spatial distribution of the data become important for intensifying health promotion and protection strategies to reduce the pathology's occurrence.
\end{abstract}

KEYWORDS: Leprosy, Incidence, Epidemiology. Spatial distribution. 


\section{Introdução}

A hanseníase é uma patologia infectocontagiosa, originada pelo Mycobacterium leprae, conhecida nos primórdios da humanidade como "lepra". Tal morbidade caracteriza-se pelo surgimento de lesões assintomáticas e anestésicas na pele, manchas pouco pigmentadas e nódulos avermelhados, além de comprometer os nervos periféricos. Sua transmissibilidade ocorre de pessoa para pessoa por meio respiratório.1-4

Tal patologia compõe a lista das outras 35 , de notificação compulsória e de investigação obrigatória no Brasil, por meio do preenchimento da ficha de notificação do Sistema de Informações de Agravo de Notificação (SINAN), após o diagnóstico da enfermidade. Em nível nacional, a hanseníase classifica-se em: virchowiano, tuberculóide, indeterminado e dimorfo. Vale considerar que as duas primeiras classificações são consideradas estáveis, enquanto as outras se configuram de forma instável. . $-7^{-7}$

No Brasil, a hanseníase continua a ser um importante problema de saúde pública, acometendo principalmente jovens e adultos em idade produtiva, ocasionando elevados custos, principalmente no de reabilitação. Apesar de o país ter assumido desde o ano de 1991 o compromisso de erradicar essa patologia até o ano 2000, as metas para tal objetivo não foram alcançadas; novas metas foram traçadas para o período de 2006 a 2010.,9

No ano de 2011, o Ministério da Saúde, por meio do Plano Integrado de estratégias de eliminação da hanseníase e outras patologias, idealizou como objetivo, até o ano de 2015, promover o desenvolvimento e a implementação de políticas públicas integradas e interprogramáticas efetivas e baseadas em evidências para a redução da carga das doenças em eliminação. Para se alcançar o objetivo relacionado à hanseníase, traçaram-se as seguintes metas: alcançar prevalência de menos de um caso para 10.000 habitantes; alcançar e manter o percentual de $90 \%$ de cura nas coortes de casos novos de hanseníase até 2015; aumentar a cobertura de exames de contatos intradomiciliares para $\geq 80 \%$ dos casos novos de hanseníase até 2015; reduzir em 26,9\% o coeficiente de detecção de casos novos de hanseníase em menores de 15 anos até 2015. 9

Em 2016, o Ministério da Saúde, por meio das Diretrizes para vigilância, atenção e eliminação da hanseníase como problema de saúde pública, estabeleceu as seguintes ações para minimização desta patologia: educação em saúde, investigação epidemiológica para o diagnóstico oportuno de casos, tratamento até a cura, prevenção e tratamento de incapacidades, vigilância epidemiológica, exame de contatos, orientações e aplicação de Bacillus Calmette-Guérin (BCG). 10

Entre os anos de 2012 a 2016, foram notificados 151.764 casos novos de hanseníase no país, correspondendo a uma incidência média de 14.97 casos novos para cada 100 mil habitantes. Destes, 84.447 casos novos foram entre a população masculina. ${ }^{11}$ No estado da Bahia, no ano de 2016, 2.060 casos novos da referida patologia foram registrados, correspondendo a uma taxa de incidência de 13,48/100.000 habitantes.12 Em Feira de Santana, para o mesmo ano, foram notificados 268 casos novos, tendo um aumento importante quando comparado aos anos anteriores. ${ }^{13}$ Feira de Santana constitui a lista dos 12 municípios prioritários do estado da Bahia para o controle da hanseníase. ${ }^{14}$ Desta forma, o presente estudo se justifica pela importância de conhecer o perfil epidemiológico e a distribuição espacial dos casos novos dessa patologia no município como forma de contribuir para ações de promoção e proteção à saúde com maior êxito, além de firmar a importância da descentralização da assistência e a implantação dessas ações nas Unidades de Saúde da Família.

Objetivou-se com este estudo descrever o perfil epidemiológico e espacial dos casos novos de hanseníase em Feira de Santana-Bahia entre os anos de 2005-2015. 


\section{Metodologia}

Trata-se de um estudo epidemiológico, descritivo, de série histórica, realizado no Município de Feira de Santana, no período de 2005 a 2015. O Município de Feira de Santana encontra-se situado a 107 km da capital baiana. É a segunda cidade mais populosa do estado da Bahia e a maior cidade do interior nordestino. Conforme o censo demográfico do Instituto Brasileiro de Geografia e Estatística (IBGE), em 2010 a população era de 556.642 habitantes. $\cdot \frac{15}{}$

Para a elaboração deste estudo, utilizou- se dados secundários obtidos no Sistema de Informação de Agravos de Notificação disponibilizados em forma de tabelas impressas pela secretaria municipal de saúde de Feira de Santana constituídas de todos os casos novos de hanseníase notificados, no período de 2005 a 2015, no município de Feira de Santana-Bahia.

As variáveis selecionadas para análise foram: sexo, cor/raça (referida), faixa etária, escolaridade, forma clínica e incapacidades dos portadores de hanseníase. As informações foram sistematizadas em tabelas, com auxílio do Programa Excel Microsoft Corporation (2007), seguindo a sequência e distribuição das variáveis do estudo.

A incidência média da hanseníase nos bairros e distritos rurais foi calculada conforme apresentado a seguir:

Incidência média $=\left(N^{\circ}\right.$ casos novos de Hanseníase $\left./ 10\right) \times 100.00$ hab.

População do bairro ou distrito rural pelo (Censo 2010)

Após o cálculo da incidência média dos casos, os dados foram armazenados em ambiente de Sistema de Informação Geográfica por meio do software Qgis, versão 2.18. Em seguida foi confeccionado um mapa coroplético, por meio da classificação Quantil, para representar a distribuição espacial da incidência média dos casos novos de hanseníase em Feira de Santana - Bahia.

Para este estudo, não foi necessária submissão do trabalho ao Comitê de Ética e Pesquisa, conforme o art. $1^{\circ}$ da Resolução Conselho Nacional de Saúde n 510/2016.

\section{Resultados}

Foram notificados no período de 2005 a 2015, no município de Feira de Santana, 1.239 casos novos de hanseníase. Destes casos, 53\% corresponderam ao sexo masculino. A faixa etária com maior frequência dos casos foi entre os indivíduos com idade entre 35 - 49 anos. Em relação à raça/cor, as pessoas que se autodeclararam de raça/ cor preta ou parda, apresentaram os maiores percentuais da afecção. No que se refere à escolaridade, 37,3\% dos indivíduos com hanseníase tinham o ensino fundamental incompleto (Tabela 1). 
Tabela 1. Características Epidemiológicas dos casos de Hanseníase em Feira de Santana - BA, 2005 a 2015

\begin{tabular}{|c|c|c|}
\hline Características & $\mathrm{N}=1239$ & $\%$ \\
\hline \multicolumn{3}{|l|}{ Sexo } \\
\hline Masculino & 658 & $53 \%$ \\
\hline Feminino & 581 & $47 \%$ \\
\hline \multicolumn{3}{|l|}{ Raça } \\
\hline Ignorado/Branco & 27 & $2 \%$ \\
\hline Branca & 99 & $8 \%$ \\
\hline Preta & 312 & $25 \%$ \\
\hline Parda & 801 & $65 \%$ \\
\hline \multicolumn{3}{|l|}{ Idade } \\
\hline $1--4$ & 4 & $0,5 \%$ \\
\hline $5--9$ & 22 & $1,8 \%$ \\
\hline $10--14$ & 51 & $4,0 \%$ \\
\hline 15--19 & 69 & $5,5 \%$ \\
\hline 20--34 & 314 & 25,5 \\
\hline 35--49 & 336 & $27 \%$ \\
\hline $50--64$ & 261 & $21 \%$ \\
\hline 65--79 & 156 & $12,5 \%$ \\
\hline $80+$ & 26 & $2,1 \%$ \\
\hline \multicolumn{3}{|l|}{ Escolaridade } \\
\hline Ignorado/Branco & 157 & $12,7 \%$ \\
\hline Analfabeto & 72 & $5,8 \%$ \\
\hline Ensino fundamental incompleto & 463 & $37,3 \%$ \\
\hline Ensino fundamental completo & 151 & $12,2 \%$ \\
\hline Ensino médio incompleto & 111 & $9.0 \%$ \\
\hline Ensino médio completo & 218 & $17,6 \%$ \\
\hline Educação superior incompleta & 14 & $1,0 \%$ \\
\hline Educação superior completa & 43 & $3,5 \%$ \\
\hline Não se aplica & 10 & $0,8 \%$ \\
\hline
\end{tabular}

Fonte: SMS, 2018

Elaborado pelos autores, 2018.

Em relação às características clínico-epidemiológicas, 33\% dos casos foram na forma dimorfa. No que se refere ao grau de incapacidade resultante desta patologia, 19,6\% apresentaram incapacidade Grau 1, enquanto $63 \%$ tiveram Grau 0 (Tabela 2).

Tabela 2. Característica Clinica e Epidemiológica dos casos de Hanseníase em Feira de Santana - BA, 2005 a 2015

\begin{tabular}{lcc}
\hline \multicolumn{1}{c}{ Características } & $\mathbf{N = 1 2 3 9}$ & \% \\
\hline Forma clinica & & $3,6 \%$ \\
Ignorado/Branco & 45 & $16.0 \%$ \\
Indeterminada & 198 & $26,4 \%$ \\
Tuberculóide & 327 & $33 \%$ \\
Dimorfa & 410 & $20,1 \%$ \\
Vichowiana & 250 & $0,72 \%$ \\
Não classificada & 9 & $0,64 \%$ \\
Grau de Incapacidade & & $63 \%$ \\
Ignorado/Branco & 8 & $19,6 \%$ \\
Grau zero & 780 & $6,6 \%$ \\
Grau 1 & 243 & $10,1 \%$ \\
Grau 2 & 82 & \\
Não avaliado & 126 & \\
\hline
\end{tabular}

Fonte: SMS, 2018

Elaborado pelos autores, 2018 
A Figura 1 mostra a distribuição espacial da incidência média dos casos novos de hanseníase. No período estudado, os bairros com as maiores incidências foram Tomba, Brasília, Calumbi, Gabriela, Campo Limpo, Mangabeira. Com relação aos distritos rurais, os que tiveram a maior incidência média foram o de Bonfim da Feira e Humildes.

Figura 1. Distribuição Espacial da Incidência média dos casos novos de Hanseníase em Feira de Santana -Bahia, entre os anos de 2005 -2015

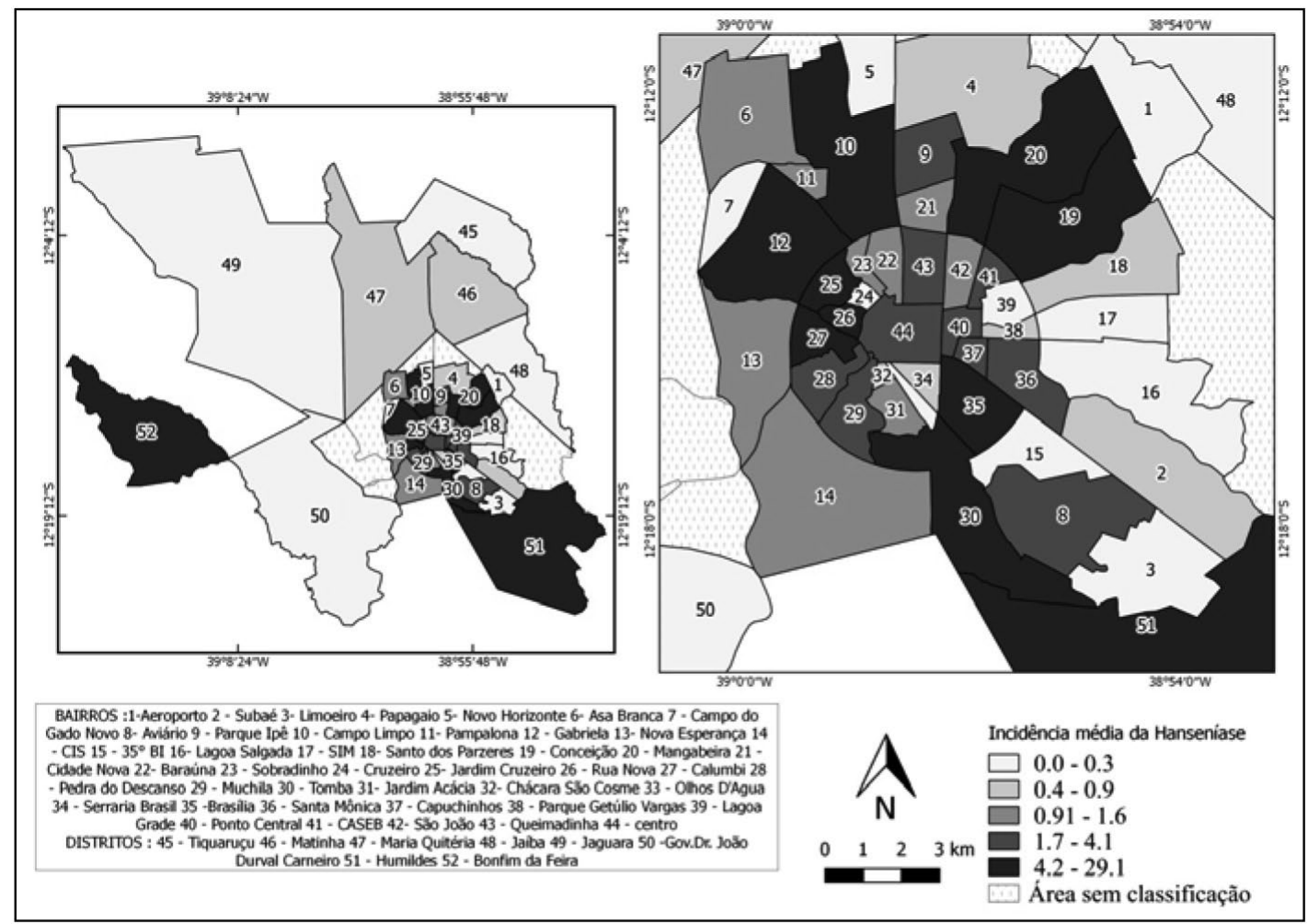

Projeção Universal Transversa de Mercator /Origem da Kilometragem: Equador e Meridiano 39 WGr/ Sistema Geodésio Sirgas 2000, Escala 1:100.000.000. Fonte: IBGE,2010; SMS,2018. Elaborado por Roquenei da Purificação Rodrigues, 2018.

\section{Discussão}

O presente estudo permitiu identificar o comportamento epidemiológico e espacial da Hanseníase em Feira de Santana- Bahia, cujo município integra a lista do plano nacional de mobilização e intensificação das ações para a eliminação da hanseníase e controle da tuberculose, estabelecido pelo Ministério da Saúde. .5 controle desta afecção envolve atividades de educação em saúde, investigação epidemiológica para o diagnóstico oportuno de casos, tratamento até a cura, prevenção e tratamento de incapacidades, vigilância epidemiológica, exame de contatos, orientações e aplicação de BCG. 10

Com relação aos casos de hanseníase entre os sexos, a maior frequência destes ocorreu entre os indivíduos do gênero masculino. De um modo geral, os homens são mais susceptíveis ao adoecimento, principalmente às infectocontagiosas, visto que possuem pouca ou nenhuma preocupação com a sua saúde. Neste contexto, Melão et al. (2011) $)^{16}$ destacam que os homens têm maior contato social entre homens, menor preocupação com o corpo e com a estética quando comparado às mulheres, que contam com programas de saúde específicos voltados para a saúde feminina. Tais fatores podem retardar o diagnóstico não só da hanseníase como de outras afecções.

No quesito raça/cor autorrefererida, o maior percentual dos indivíduos foram os pardos. Há de se considerar que conforme o último Censo Demográfico (2010) realizado pelo Instituto Brasileiro de Geografia e Estatística, 55.84\% da população feirense declarou-se parda. Aquino et al. (2003)17 destacam que na região Nordeste do Brasil existe uma proporção da população autodeclarada parda, devido ao processo de miscigenação. 
Quanto à faixa etária, o estudo demonstrou que o maior percentual dos casos ocorreu entre os indivíduos entre 35 a 49 anos, seguido daqueles com 20 a 34 anos. De acordo com Miranzi et al. (2010)18, a população economicamente ativa é a mais afetada pela hanseníase, o que pode prejudicar a economia do município, visto que essa faixa da população pode vir a desenvolver incapacidades, lesões, estados reacionais, afastar-se da atividade produtiva e gerar um custo social demasiado.

No que concerne ao grau de escolaridade, as pessoas com ensino fundamental incompleto foram as que apresentaram a maior frequência da patologia, seguido daquelas que não possuíam qualquer grau de instrução. Tal achado corrobora com o estudo de Araújo et al. (2017) ${ }^{19}$, em que $50,93 \%$ tinham ensino fundamental incompleto e $11,11 \%$ eram analfabetos. É importante considerar que indivíduos com baixo nível de instrução são mais suscetíveis a desenvolver patologias, visto que existe uma resistência à educação em saúde, a não prosseguir o andamento do tratamento ou dificuldade em compreender os receituários. $\underline{\underline{20}}$

No que se refere à apresentação da patologia, a forma dimorfa predominou. Diversos estudos realizados no Estado da Bahia têm encontrado a maior predominância em tal forma. . $0-22,24$ Costa (2017) $)^{24}$, ao caracterizar as questões epidemiológicas da hanseníase no Estado da Bahia, destaca que esta é a forma mais comum da doença e, devido ao diagnóstico tardio e as condições sanitárias, é a que mais se inocula.

Neste estudo, o maior número de pessoas não apresentou qualquer tipo de incapacidade relacionada à patologia. Alves etal. (2017) $\frac{25}{}$ destacam que a avaliação do grau de incapacidade física auxilia na estimativa da situação epidemiológica e é utilizada como indicador de controle da hanseníase. A sua detecção indica o diagnóstico tardio da doença. As incapacidades físicas e deformidades decorrentes da hanseníase constituem um problema de grande magnitude, visto que, a maioria dos indivíduos está em fase produtiva. Desta forma, os desenvolvimentos das incapacidades podem comprometer as atividades laborais, ocasionando perdas econômicas, sociais e psicológicas, podendo afetar a qualidade de vida destes. $\underline{\underline{26}}$
Este é o primeiro estudo em que foi realizada a espacialização dos dados dos casos novos de hanseníase em Feira de Santana - Bahia. Os resultados apontaram que a distribuição desses se dá em sua grande maioria nos bairros periféricos. No estudo realizado por Moura et al. $\stackrel{27}{ }$, realizado em Juazeiro - Bahia, verificou-se que houve o maior número de casos novos da hanseníase nos bairros periféricos. Tal evento também foi verificado por Bastos (2017) ${ }^{28}$, em Palmas - Tocantins.

Neste contexto é importante enfocar que não só a hanseníase quanto outras patologias infectocontagiosas tendem a se disseminar nas periferias das grandes cidades, visto que, são zonas de alta densidade de concentração humana, com precárias condições básicas de vida. $\underline{29}$

\section{Conclusão}

Por ser um estudo com dados secundários, a limitação do estudo consistiu pelo número de dados não preenchidos. Todavia, evidenciou-se que a hanseníase em Feira de Santana, no período estudado, foi caracterizada principalmente em indivíduos do sexo masculino e em pessoas com idade produtiva e com baixa escolaridade. Com relação à distribuição espacial, verificou-se que os bairros periféricos apresentaram o maior número de casos. Tal informação tornase importante para intensificação de estratégias de promoção e proteção à saúde, a fim de se reduzir a ocorrência da patologia nestas regiões.

\section{Contribuições dos autores}

Todos os autores se responsabilizaram pela elaboração do desenho de estudo, análise e interpretação dos resultados, e redação do manuscrito.

\section{Conflitos de interesses}

Nenhum conflito financeiro, legal ou político envolvendo terceiros (governo, empresas e fundações privadas, etc.) foi declarado para nenhum aspecto do trabalho submetido (incluindo, mas não se limitando a subvenções e financiamentos, participação em conselho consultivo, desenho de estudo, preparação de manuscrito, análise estatística, etc.). 


\section{Referências}

1. Ribeiro MDA, Silva JCA, Oliveira SB. Estudo epidemiológico da hanseníase no Brasil: reflexão sobre as metas de eliminação. Rev Panam Salud Publica. 2018;42:e42. https://doi.org/10.26633/ RPSP.2018.42

2. Arantes CK, Garcia MLR, Filipe MS, Nardi SMT, Paschoal VD. Avaliação dos serviços de saúde em relação ao diagnóstico precoce da hanseníase. Epidemiol Serv Saude [Internet]. 2010;19(2):155-64. Disponível em: http://scielo.iec.gov.br/pdf/ess/ v19n2/v19n2a08.pdf

3. Santos DAS, Spessatto LB, Melo LS, Olinda RA, Lisboa HCF, Silva MS. Prevalência de casos de hanseníase. Rev enferm UFPE on line [Internet]. 2017;11(Supl. 10):4045-55. Disponível em: https://periodicos.ufpe.br/revistas/revistaenfermagem/article/ viewFile/231164/25125

4. Santos AS, Castro DS, Falqueto A. Fatores de risco para transmissão da Hanseníase Fatores de risco para transmissão da Hanseníase. Rev Bras Enferm. 2008;61(esp):738-43. http://dx.doi. org/10.1590/S0034-71672008000700014

5. Portaria $n^{\circ} 1.943$, de 18 outubro de 2001 (Brasil). Define a relação de doenças de notificação compulsória para todo território nacional [Internet]. Diário Oficial da da União. 2001 out 24. Disponível em: http://scielo.iec.gov.br/pdf/iesus/v10n1/ v10n1a07.pdf

6. Ministério da Saúde (Brasil), Secretaria de Políticas de Saúde, Departamento de Atenção Básica. Guia para o Controle da Hanseníase [Internet]. Brasília: Ministério da Saúde; 2002. Disponível em: https://bvsms.saude.gov.br/bvs/publicacoes/ guia_de_hanseniase.pdf

7. Beiguelman B. Genética e Hanseníase. Ciênc. saúde coletiva [Internet]. 2002;7(1):117-28. Disponível em: https://scielosp.org/ $\mathrm{pdf} / \mathrm{csc} / 2002 . \mathrm{v} 7 \mathrm{n} 1 / 117-128 / \mathrm{pt}$

8. Penna GO, Domingues CMAS, Siqueira Júnior JB, Elkhoury ANSM, Cechine MP, Grossi MAF, et al. Doenças dermatológicas de notificação compulsória no Brasil. An Bras Dermatol. 2011;86(5):865-77. https://doi.org/10.1590/S036505962011000500002

9. Ministério da Saúde (Brasil), Secretaria de Vigilância em Saúde, Departamento de Vigilância em Doenças Transmissíveis. Plano integrado de ações estratégicas de eliminação da hanseníase, filariose, esquistossomose e oncocercose como problema de saúde pública, tracoma como causa de cegueira e controle das geohelmintíases: plano de ação 2011-2015 [Internet]. Brasília: Ministério da Saúde; 2013. Disponível em: http://bvsms.saude. gov.br/bvs/publicacoes/plano_integrado_acoes_estrategicas hanseniase.pdf
10. Ministério da Saúde (Brasil), Secretaria de Vigilância em Saúde, Departamento de Vigilância das Doenças Transmissíveis. Diretrizes para vigilância, atenção e eliminação da Hanseníase como problema de saúde pública [Internet]. Brasília: Ministério da Saúde, 2016. Disponível em: https://portalarquivos2.saude.gov.br/ images/pdf/2016/fevereiro/04/diretrizes-eliminacao-hanseniase4fev16-web.pdf

11. Secretaria de Vigilância em Saúde. Caracterização da situação epidemiológica da hanseníase e diferenças por sexo, Brasil, 2012-2016. Boletim Epidemiológico [Internet]. 2018;49(4):1-12. Disponível em: https://antigo.saude.gov.br/images/pdf/2018/ fevereiro/19/2018-004-Hanseniase-publicacao.pdf

12. Governo do Estado da Bahia, Secretaria da Saúde. Boletim epidemiológico hanseníase [Internet]. Bahia: Governo do Estado; 2017. Disponível em: http://www.saude.ba.gov.br/wp-content/ uploads/2017/11/Boletim_epidemiologico_hanseniase_2017.pdf

13. Ministério da Saúde (Brasil), Departamento de Informática do Sistema Único de Saúde. Acompanhamento dos dados de hanseníase - Bahia [Internet]. [acesso em 2018 ago. 28]. Disponível em: http://tabnet.datasus.gov.br/cgi/tabcgi. exe?sinannet/cnv/hanswbr.def

14. Ministério da Saúde (Brasil), Secretaria de Vigilância em Saúde. Sistema Nacional de Vigilância em Saúde: relatório de situação: Bahia [Internet]. Brasília: Ministério da Saúde; 2005. Disponível em: http://bvsms.saude.gov.br/bvs/publicacoes/ba1.pdf

15. IBGE Cidades. Brasil: IBGE; 2017. Disponível em: $\underline{\text { https:// }}$ cidades.ibge.gov.br/

16. Melão S, Blanco LF, Mounzer N, Veronezi CC, Simões PW. Perfil epidemiológico dos pacientes com hanseníase no extremo sul de Santa Catarina, no período de 2001 a 2007. Rev Soc Bras Med Trop. 2011;44(1):79-84. https://doi.org/10.1590/S003786822011000100018

17. Aquino DMC, Caldas AJM, Silva AAM, Costa JML. Perfil dos pacientes com hanseníase em área hiperendêmica da Amazônia do Maranhão, Brasil. Rev Soc Bras Med Trop. 2003;36(1):57-64. https://doi.org/10.1590/S0037-86822003000100009

18. Miranzi SSC, Pereira LHM, Nunes AA. Perfil epidemiológico da hanseníase em um município brasileiro, no período de 2000 a 2006. Rev Soc Bras Med Trop 2010; 43(1):62-7. https://doi. org/10.1590/S0037-86822010000100014

19. Araújo AERA, Aquino DMC, Goulart IMB, Pereira SRF, Figueiredo IA, Serra HO, et al. Complicações neurais e incapacidades em hanseníase em capital do nordeste brasileiro com alta endemicidade. Rev bras epidemiol. 2014;17(4):899-910. https://doi.org/10.1590/1809-4503201400040009 
20. Freitas DV, Xavier SS, Lima MAT. Perfil Epidemiológico da hanseníase no município de Ilhéus-BA, no período de 2010 a 2014. J health Sci. 2018;19(4):274-7. https://doi. org/10.17921/2447-8938.2017v19n4p274-277

21. Souza C, Rodrigues M. Magnitude, tendência e espacialização da hanseníase em menores de 15 anos no estado da Bahia, com enfoque em áreas de risco: um estudo ecológico. Hygeia [Internet]. 2015;11(20):201-22. Disponível em: http://www.seer. ufu.br/index.php/hygeia/article/view/28914

22. Santos $A D$, Santos MB, Barreto AS, Carvalho DS, Alves AB, Araújo KCGM. Análise espacial e características epidemiológicas dos casos de hanseníase em área endêmica. Rev. enferm UFPE on line [Internet]. 2016;10(5):4188-97. Disponível em: https:// pesquisa.bvsalud.org/portal/resource/pt/bde-29991

23. Santana JC, Santos MC, Lima MATL, Carvalho LR. Perfil Epidemiológico da hanseníase em Itabuna - Bahia. J. nurs. health. 2018;8(2): e188206. https://doi.org/10.15210/jonah.v8i2.13143

24. Costa AKAN. Características epidemiológicas da hanseníase no estado da Bahia, 2005 - 2015 [dissertação] [Internet]. Goiânia (GO): PUC Goiás; 2017. Disponível em: http://tede2.pucgoias.edu. br:8080/handle/tede/3724

25. Alves ES, Oliveira LB, Araújo TME, Melo IV, Araújo RPS, Marques LMF. Perfil epidemiológico da hanseníase em um município do nordeste brasileiro: uma análise retrospectiva. J res fundam care online [Internet]. 2017;9(3):648-52. Disponível em: https://www.redalyc.org/pdf/5057/505754116007.pdf
26. Amaral EP, Lana FCF. Análise espacial da Hanseníase na microrregião de Almenara, Rev. Bras. Enferm. 2008;61(esp):701-7. https://doi.org/10.1590/\$0034-71672008000700008

27. Moura LTR, Fernandes TRMO, Bastos LDM, Luna ICF, Machado LB. Hanseníase em menores de 15 anos na cidade de JuazeiroBa. Rev. Hansenol int. 2012;37(1):45-50. Disponível em: http:// periodicos.ses.sp.bvs.br/scielo.php?script=sci_arttext\&pid=S198251612012000100005\&lng=pt\&nrm=iso\&tlng=pt

28. Bastos WM. Características sociodemográficas e epidemiológicas da hanseníase do município de palmas - Tocantins [dissertação] [Internet]. Salvador (BA): Universidade Federal do Estado da Bahia; 2017. Disponível em: https://repositorio.ufba.br/ri/bitstream/ri/25732/1/ DISSERTA\%C3\%87\%C3\%830\%20FINAL\%20MP.\%20Whisllay\%20 Bastos.\%202017.pdf

29. Ministério da Saúde (Brasil), Secretaria de Atenção à Saúde, Departamento de Atenção Básica. Vigilância em Saúde: Dengue, Esquistossomose, Hanseníase, Malária, Tracoma e Tuberculose [Internet]. Brasília: Ministério da Saúde; 2008. Disponível em: http://bvsms.saude.gov.br/bvs/publicacoes/cab_n21_vigilancia_ saude_2ed_p1.pdf 